\title{
Using Twitter to Extend Your Department's Outreach and Visibility
}

\author{
Lucie S. Ciccone', George A. Van den Driessche², and Maria T. Gallardo-Williams² \\ ${ }^{1}$ Departments of Biological Sciences and ${ }^{2}$ Chemistry, North Carolina State University, Raleigh, NC \\ Keywords: Chemistry Outreach, Twitter, Recruitment, Departmental Visibility, Social Media \\ Publication Date: May 3, 3021
}

\begin{abstract}
Social media platforms like Twitter offer benefits for promoting and sharing scientific research. Each user becomes an instant news source for their work, and are able to report lab updates, news, and discoveries in near real time, increasing the visibility and citations of one's research and allowing for direct and public engagement with the scientific community. Additionally, such outreach has the possibility of strengthening collaboration and engagement within one's academic department. However, amidst the noise of a billion profiles, how can you be heard and measure your impact? The North Carolina State University Department of Chemistry has adopted an amplification method that relies on promoting departmental news through collaboration between faculty (@NCStateChem) and student organization (@NCStateChemGSA) run accounts, which are then amplified through research lab or personal community member profiles. Because of this strategy, our Twitter account has a following that extends beyond our own students and faculty. In fact, nearly three quarters of our Twitter follower base consists of accounts not affiliated with NC State. Here we share insights into one of our social media strategies for Twitter (\#ChemPack) and provide an overview of our in-house amplification network monitoring impressions and engagement rate as a metric to assess our impact.
\end{abstract}

\section{INTRODUCTION}

Researchers and academic departments face the constant challenge of creating research with high visibility that establishes academic credibility in the scientific community and, in some instances, is required for procuring research funding and for the job placement success of the students that graduate from the department (Hadani et al., 2012). Historically, the visibility of academic chemistry departments was largely based on the number and quality of publications produced, and promoting these publications occurred through personal networks, invited talks, or news coverage (Burris, 2004; Metzger and Kallio, 2018). Often, such strategies are limited and can even be exclusionary in practice, as they ignore differences between disciplines, reinforce academic hierarchies based on privilege and visibility (and equally, invisibility), and focus on promoting individual researchers rather than collective departments. Some members of a department might be more likely to cultivate their individual visibility, which might in turn reflect on their home institution, but this approach can be slow and highly dependent on the continuation of the individual's appointment in the department (Leahey, 2007; Mauvais-Jarvis, 2012). Many universities have made investments in improving the impacts of their online presence, and a positive correlation has been found between a school's web visibility and their rankings (Lee and Park, 2012). In addition to online efforts, in-person events, such as recruitment meetings, conferences, or networking events, are common tools academic departments use to promote their brand within their institution and beyond. While undeniably valuable, these events are often limited by cost and scope.

The capabilities of the internet, however, are rapidly changing our societal understanding of, and relationship to, communication, information, and outreach. As the internet continues to develop as a widespread, primary information source for many, it is becoming increasingly expected for individuals and organizations in academia to develop a professional online presence as a form of outreach. Social media is one facet of this and is beginning to be incorporated into academic and professional communities. Through its versatility and accessibility, microblogging platforms such as Twitter have the potential to be used as a novel, cheap, and inclusive medium through which academic departments and researchers can extend their visibility and to reach more diverse audiences. Additionally, online social media platforms 
can also serve as a forum for collaboration, encouragement, teaching, and building a sense of community while inspiring mutual growth and visibility (Prabhu and Rosenkrantz, 2015; Ciprut et al., 2017; Bodnar and Fox, 2019).

Addressing Inequitable Visibility in Academic Settings. Within academia, the ways of establishing departmental visibility that are commonly thought of as "traditional"number of publications, reputation of certain faculty members - can too often rely on word-of-mouth promotions and a culture of exclusivity. Additionally, such approaches are often focused on promoting individual faculty member researchers, rather than the department as a whole. In general, these approaches also ignore the issue of cultural and gender taxation, by which certain work such as teaching, mentoring, and serving on committees is feminized and undervalued. This kind of work frequently goes unrecognized and often unduly falls upon women or faculty of color who, in turn, have less time to dedicate themselves to activities that might bring them academic success and visibility (Riegraf and Weber, 2017; Social Sciences Feminist Network Research Interest Group, 2017).

The development and spread of the internet has made it possible, if given access to a computer and the internet, for anyone regardless of fame or status to publish and promote their work through blogs, forums, and social media platforms. This indiscriminate accessibility gives social media platforms, such as Twitter, the potential to disrupt antiquated practices of determining visibility. Social media is powerful in that it provides a space where equity is possible: anyone has the ability to voice their opinion or to participate and impact the conversations of a certain community, and by using this in an intentional way, departments can elevate not just certain faculty within a department, but the department as a whole. In this way, using Twitter is not just valuable as an attempt to stay "up to date" or "on-trend", but instead can be an intentional, active medium to elevate those underrepresented in academia (Yammine et al., 2018).

The role that social media can play in elevating the perceived visibility of an academic department, and, in the process, that of its faculty and students cannot be underestimated. Strategic use of a free resource can result in better outcomes and stronger impacts for all the participants and can pave the way for the future success of the department's graduates, who will be empowered to use the same techniques in their future endeavors.

\section{PROGRAM DESCRIPTION}

The North Carolina State University Department of Chemistry seeks to use Twitter as such a platform. Here we describe the ways in which the North Carolina State University Department of Chemistry Communication Committee uses Twitter as a medium for elevating the department by focusing on a case study: the department's annual graduate student recruitment weekend, \#ChemPack. By employing a "triangulation" amplification approach through which the Twitter posts of the department Twitter account (@NCStateChem) are shared by partner accounts, as well as through the use of targeted hashtags and content, the NC State Department of Chemistry has been able to expand its reach to tens of thousands of Twitter users. At the same time, by using Twitter to highlight each lab in the department, the department seeks to intentionally and consciously celebrate all members of the department, not just some.

ChemPack is an annual recruitment event for admitted candidates to the NC State Chemistry graduate program where recruits are brought to NC State's campus for a weekend. Over the course of the weekend recruits are able to attend a department poster session, tour the campus and facilities, and meet with faculty that they are interested in working with.

Starting in 2019, the NC State Department of Chemistry began an extended, yearly content series on Twitter surrounding ChemPack in order to promote the event, raise awareness about the opportunities and faculty present in the department, and welcome recruits to the departmental community. Each week in the months leading up to the in-person recruitment weekend a different lab within the department was highlighted through a series of posts created by members of the lab. These posts introduced faculty and student members of the lab, described current projects, highlighted events, and promoted the lab and its members by tagging its members and using hashtags. Each post in the series was tagged with the hashtag \#ChemPack. Other hashtags popular on Twitter, such as \#RealTimeChem, were also used in order to further amplify this content. During the recruitment weekend itself, Twitter engagement among attendees was encouraged through several Twitter engagement giveaways.

To amplify their Twitter content, the NC State Department of Chemistry Communications Committee has developed a system of "triangulation". In this system, the Twitter content created by the department and Chemistry Graduate Student Association (@NCStateChemGSA) Twitter accounts is mutually retweeted by each other and by the faculty and students in the department. By posting and retweeting content from multiple accounts, the Department of Chemistry is able to increase the frequency at which its content is promoted in users' Twitter timelines. This content is further promoted by informal partnerships that the Department of Chemistry has actively sought to develop between the department account and graduate students, faculty, and other on-campus organizations and departments active on Twitter. Each of these partner accounts have different pools of followers, and as a result, when content of the department is retweeted by these accounts, it is able to reach a wider and more diverse audi- 
ence. The Department of Chemistry Communications committee, via the Twitter account, has been able to build these relationships by making an effort to engage with and retweet the content of the department's partners, and as a result of this reciprocity both the department and the department's partners benefit. Because these partnerships are loose and informal, the success of content is not assured, and much of it has to do with chance (who sees it), and the responses of those that do, which vary unpredictably. However, by intentionally connecting with student and faculty groups across campus, through this model the department is able to extend and diversify their reach and, in the process, to strengthen collaborative relationships with faculty, students, and groups both within and outside of the department.

\section{EVALUATION METHODS}

To assess the impact of employing these techniques, the NC State Department of Chemistry Communications Committee used Twitter Analytics and open source tools to gather data on the number and frequency of engagements, impressions, followers, and retweets with department and CGSA content. This data was collected monthly starting in 2018 and charted over time.

Twitter Analytics. Twitter Analytics is a built-in content analysis program provided by Twitter that allows a Twitter user to see the number and frequency of impressions (i.e. views) and engagement (i.e. retweets, replies, and likes) that their content has received. This data is available for the entire time a user's account is active, and can be downloaded as an Excel file. Data for the NC State Department of Chemistry and Graduate Student Association Twitter accounts were downloaded for month-long periods and analyzed.

Open Source Tools. The open source twitteR package was used to download tweets from other user accounts in order to analyze the number and content of related tweets. By providing access to the Twitter API, twitteR allows one to download, clean, and analyze tweets from a user or hashtag from the past 7 days. Unlike Twitter Analytics, the twitteR package does not record the views or impressions per tweet, but allows one to assess what Twitter users view, retweet, or use a specific hashtag. Tweets posted by @NCStateChem,@NCStateCGSA, and other accounts that used the hashtag \#ChemPack were downloaded. The words included in these tweets were counted and visualized using WordMaps, a text-mining visualization that uses relative size to indicate the frequency that different words are used. These word counts, represented through such visualizations as WordMaps, give insight into what topics users--in particular students--considered most important and relevant to ChemPack.

\section{RESULTS}

Analysis of Department and CGSA Accounts. Prior to the launch of the ChemPack 2019 Twitter campaign, analysis was conducted of the Department and CGSA Twitter accounts from June through October 2018 using Twitter Analytics and twitteR. This analysis revealed the positive impact of using targeted Twitter content and collaborating with student, faculty, and organizational partners on Twitter engagement and departmental reach.

During October 2018, the CGSA account hosted a "graduate student take-over" in which the account was run by a graduate student for a period of four days. Over the course of the week this student published 36 tweets focused on daily topics including her experiences with chemistry research, being a woman in STEM, chemistry mentorship, and why she chose NC State. As a result of this content, the CGSA account received 6 times the number of impressions in October as in September 2018, and the average number of impressions per Tweet increased by 1.67. This increase in engagement with the CGSA account demonstrates the potential success of launching targeted, short Twitter campaigns at reaching new audiences and communicating about the work of the department and what it is like to study chemistry. It also shows the importance of developing collaborative relationships between faculty and student Twitter users.

The success of these intentional Twitter efforts provided much of the impetus for the development of the ChemPack Twitter campaign in 2019. The combination of tracking historical social media analytics and running lab featured campaigns resulted in the efforts to create a social media campaign during recruitment weekend. The data from these efforts provided the department with a tangible measure of the value of social media.

\#ChemPack2019 Campaign. In preparation for the \#ChemPack2019 campaign, all triangulation partners were alerted via email of the upcoming effort, and their voluntary collaboration in the form of likes and retweets was requested. In addition, members of the Communications Committee created a series of 20 tweets highlighting features of the Department that were considered attractive to prospective students. The sub-committee that prepared the tweets was composed of faculty, staff, and graduate students with diverse backgrounds and prior levels of social media expertise, who worked together. The tweets included facts about NC State University, living in the Raleigh, NC area, the general culture of the Department, and the teaching and research facilities available to graduate students.

Analysis of Twitter data from ChemPack 2019 revealed that, over the course of the single weekend, the content of the department and CGSA accounts collectively received 47,100 impressions. The tweets that highlighted our research faculty and their interests received the most likes and retweets 


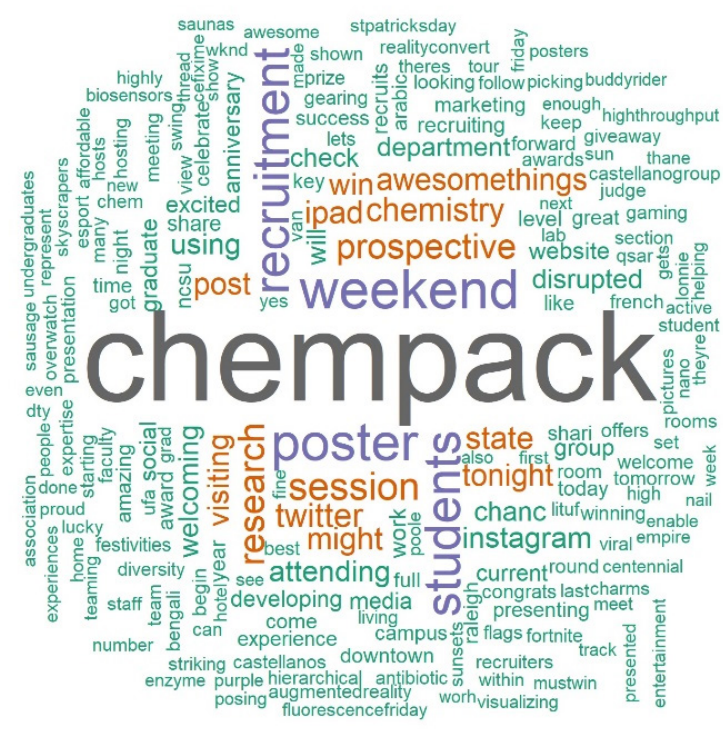

Figure 1. \#ChemPack2019 WordMap

during the campaign. Individual triangulation partners organically retweeted content that aligned with their own accounts. For example, the CGSA account was more likely to retweet content related to graduate student working conditions, and living in the area, while the individual faculty and student triangulation partners highlighted content directly related to their own labs or research interests. Additionally, WordMaps representing the content of the posts containing the hashtag "ChemPack" revealed that the most commonly used words, "weekend", "recruitment", "students", "research", were highly applicable to the goal of ChemPack, showing that the content that was created by the department and partner accounts throughout the weekend was successful in expressing targeted information (see Figure 1).

Given the large number of impressions received during the sampling period, and the limitations of the sampling methods it is not possible to describe the nature of each of the unique impressions that were a result of this campaign. However, a breakdown of the composition of the follower base of the account at the end of the sampling period could be used to describe the reach of the account beyond the Department and the University (summarized in Table 1).

When the different types of followers are quantified, it can be seen than only a small percentage of the total $(5.8 \%)$ are other official NC State accounts, other academic departments and units outside the university $(4.3 \%)$, or students and faculty of the Department (12.7 and 1.0\%, respectively). Although those constituencies are important, because it means that the account can be used to reach the native audience of @NCStateChem, they are not the bulk of the followers. Of the accounts following @NCStateChem on Twitter at the time of the sampling $72.9 \%$ were not affiliated directly or indirectly with the Department of Chemistry, and can be considered as outreach targets. Some of those accounts belonged to prospective graduate students, as well as undergraduates interested in applying to our programs. Parents and high school teachers are also part of the unaffiliated audience, and can have an important amplification effect if they engage with our content and share it with any prospective students.

While we were very pleased with the overall reach of the account based on the interactions described here, the nature of Twitter is such that most of those interactions are limited to a like, expanding a post to see a picture, or a retweet. Most users do not reply to posts unless they have specific questions, and the examples presented here are no exception. For that reason, we cannot offer a perspective on the individual reactions of the end users of the content. From our experience, the most engagement happens right after posting, and it is typically enhanced if the post is retweeted by a partner with a large number of followers.

In the five months leading up to ChemPack, individual posts highlighting labs within the department received upwards of 27,700 impressions. Since these events, faculty outside of the Department of Chemistry Communications committee have created their own Twitter and lab accounts, demonstrating that these collaborative faculty highlights were successful in both increasing the visibility of these labs and bolstering enthusiasm for departmental Twitter outreach.

\section{CONCLUSION}

The quantitative success of the ChemPack Twitter campaign (as seen through the large number of impressions) demonstrates the viability of using Twitter as a departmental platform for communication and outreach. Additionally, the positive responses received from faculty members as a result of this program indicates its success at helping to foster a sense of departmental pride and community. Through employing this type of outreach medium, people within the department whose work would otherwise not be visible to a

\begin{tabular}{lc}
\hline Type of Follower & $\begin{array}{l}\text { Percentage of Total } \\
\text { Followers } \\
(\mathbf{n = 9 1 5 )}\end{array}$ \\
\hline Other official NC State accounts & $5.8 \%$ \\
Other academic departments and units outside & $4.3 \%$ \\
NC State & $12.7 \%$ \\
NC State Chemistry students and alumni & $1.0 \%$ \\
NC State Chemistry faculty & $1.5 \%$ \\
Vendors & $1.9 \%$ \\
Publishers and chemistry journals & $72.9 \%$ \\
Non-affiliated accounts (individual accounts & \\
not connected to NC State Chemistry) & \\
\hline
\end{tabular}

Table 1. Percent composition of the follower base of the @NCStateChem account on March 31, 2019 (after the conclusion of the ChemPack Twitter campaign). 
general public were made visible.

After the successful conclusion of our ChemPack Twitter campaign in 2019 we continued our Twitter outreach efforts. Due to the effects of the COVID-19 pandemic, our recruitment weekend had to be moved to a virtual format in 2020 , and we leveraged our social media presence to reach as many prospective graduate students as possible. Even under very difficult conditions, we were successful in our recruiting efforts, and we consider that our social media strategy was a very important part of this effort.

The use of a free, simple, and accessible tool such as Twitter has enabled our academic department to engage our faculty, staff, and students, and to reach beyond to other constituencies, including alumni, other academic departments, and our own university administration. Having a large and diverse Communications Committee that included faculty, staff, and graduate students played a very important role in these developments. Twitter and other social media and microblogging platforms are increasingly being recognized as beneficial for use in teaching scientific communication, measuring scholarly impact, and providing opportunities for networking that are equitable and open to all.

\section{AUTHOR INFORMATION Corresponding Author}

Maria T. Gallardo-Williams. Maria_Gallardo@ncsu.edu

\section{Author Contributions}

The manuscript was written through contributions of all authors. All authors have given approval to the final version of the manuscript.

\section{ACKNOWLEDGMENTS}

The authors appreciate the technical support and assistance of the Department of Chemistry Communications Committee and the North Carolina State University College of Sciences Marketing and Communications Team. We also appreciate the comments received from our reviewers, which greatly strengthened this work.

\section{ABBREVIATIONS}

CGSA: Chemistry Graduate Student Association; NC: North Carolina

\section{REFERENCES}

Bodnar, L. M., and Fox, M. P. (2020). What the hashtag? Using twitter and podcasting to extend your scientific reach. Paediatric and Perinatal Epidemiology, 34, 1-3. doi:10.1111/ ppe. 12630

Burris, V. (2004). The Academic caste system: Prestige hierarchies in $\mathrm{PhD}$ exchange networks. American Sociological Review, 69(2), 239-264. doi:10.1177/000312240406900205

Ciprut, S., Curnyn, C., Davuluri, M., Sternberg, D., and Loeb, S. (2017). Twitter activity associated with US News and World Report reputation scores for urology departments. Urology, 108, 11-16. doi:10.1016/j.urology.2017.05.051

Hadani, M., Coombes, S., Das, D., and Jalajas, D. (2012). Finding a good job: Academic network centrality and early occupational outcomes in management academia. Journal of Organizational Behavior, 33, 723-739. doi:10.1002/job.788

Leahey, D. (2007). Not by productivity alone: How visibility and specialization contribute to academic earnings. American Sociological Review, 72(4): 533-561. doi:10.1177/000312240707200403

Lee, M., and Park. H. W. (2012). Exploring the web visibility of world-class universities. Scientometrics, 90, 201-218. doi:10.1007/s11192-011-0515-6

Mauvais-Jarvis, F. (2016). Developing academic visibility in the medical sciences. Ochsner Journal, 16(3), 208-209.

Metzger, J., and Kallio, K. P. (2018). "Alternative” journal publishing and the economy of academic prestige. Fennia, 196(1), 1-3. doi:10.11143/fennia.70469

Prabhu, V., and Rosenkrantz, A. B. (2015). Enriched audience engagement through Twitter: Should more academic radiology departments seize the opportunity? Journal of the American College of Radiology, 12(7) 756-759. doi:10.1016/j. jacr.2015.02.016

Riegraf, B., and Weber, L. (2017). Excellence and Gender equality policies in neoliberal universities. Gender and Research / Gender a Výzkum, 18(1), 92-112. doi:10.13060/2570657 8.2017.18.1.351

Social Sciences Feminist Network Research Interest Group. (2017). The burden of invisible work in academia: Social inequalities and time use in five university departments. Humboldt Journal of Social Relations, 39, 228-245.https:// www.jstor.org/stable/90007882

Yammine, S. Z., Liu, C., Jarreau, P. B., and Coe, I. R. (2018). Social media for social change in science. Science, 360, 162-163. doi:10.1126/science.aat7303 